\title{
Philosophiques
}

\section{La réponse naturelle : une solution inadéquate au dilemme darwinien}

\section{Félix Aubé Beaudoin}

Volume 42, numéro 1, printemps 2015

URI : https://id.erudit.org/iderudit/1032221ar

DOI : https://doi.org/10.7202/1032221ar

Aller au sommaire du numéro

\section{Éditeur(s)}

Société de philosophie du Québec

ISSN

0316-2923 (imprimé)

1492-1391 (numérique)

Découvrir la revue

Citer cet article

Aubé Beaudoin, F. (2015). La réponse naturelle : une solution inadéquate au dilemme darwinien. Philosophiques, 42(1), 131-151.

https://doi.org/10.7202/1032221ar
Résumé de l'article

Le dilemme darwinien, formulé par Sharon Street, somme les réalistes moraux d'expliquer pourquoi de nombreux jugements qui sont des candidats au statut de vérités morales indépendantes sont aussi ceux qui ont une grande valeur sélective. Les réalistes peuvent soit nier, soit affirmer l'existence d'un lien entre pressions évolutionnistes et vérités morales. Selon Street, la première option mène au scepticisme tandis que la seconde est indéfendable sur le plan scientifique. Peter Singer et Katarzyna de Lazari-Radek optent pour la première branche de ce dilemme. Dans cet article, la stratégie argumentative qu'ils adoptent - la réponse naturelle - sera soumise à un examen critique. Deux objections seront formulées. La première est d'ordre épistémologique : l'intuitionnisme philosophique défendu par les auteurs fait face à des difficultés majeures. La seconde, plus fondamentale, est que leur solution ne permet pas d'expliquer autrement que par un heureux hasard l'alignement entre les vérités morales et les jugements ayant une valeur sélective. 


\title{
Varia
}

\section{La réponse naturelle: une solution inadéquate au dilemme darwinien}

\author{
FÉLIX AUBÉ BEAUDOIN \\ Université Laval \\ felix.aube-beaudoin.1@ulaval.ca
}

\begin{abstract}
RÉSUMÉ. - Le dilemme darwinien, formulé par Sharon Street, somme les réalistes moraux d'expliquer pourquoi de nombreux jugements qui sont des candidats au statut de vérités morales indépendantes sont aussi ceux qui ont une grande valeur sélective. Les réalistes peuvent soit nier, soit affirmer l'existence d'un lien entre pressions évolutionnistes et vérités morales. Selon Street, la première option mène au scepticisme tandis que la seconde est indéfendable sur le plan scientifique. Peter Singer et Katarzyna de Lazari-Radek optent pour la première branche de ce dilemme. Dans cet article, la stratégie argumentative qu'ils adoptent - la réponse naturelle - sera soumise à un examen critique. Deux objections seront formulées. La première est d'ordre épistémologique: l'intuitionnisme philosophique défendu par les auteurs fait face à des difficultés majeures. La seconde, plus fondamentale, est que leur solution ne permet pas d'expliquer autrement que par un heureux hasard l'alignement entre les vérités morales et les jugements ayant une valeur sélective.
\end{abstract}

ABSTRACT. - According to Sharon Street's Darwinian Dilemma, moral realists must explain why many judgments that are likely to be independent moral truths are those it would be evolutionarily adaptive to hold. Realists can either deny or assert the existence of a relation between evolutionary influences and moral truths. The first option leads to skepticism, while the second is unacceptable on scientific grounds, says Street. Peter Singer and Katarzyna de LazariRadek take the first horn of this dilemma. In this article, the argumentative strategy they adopt — the Natural Reply - is submitted to critical scrutiny. Two objections are raised. The first objection is epistemological: philosophical intuitionism, as defended by the authors, is an untenable position. The second objection is that their solution must appeal to unlikely coincidences to account for the overlap between independent moral truths and evolutionarily adaptive attitudes.

\section{Introduction}

Le réalisme moral est une position importante en métaéthique. L'une de ses principales caractéristiques est de postuler l'existence de vérités morales qui sont indépendantes des attitudes évaluatives des individus. Autrement dit, la vérité d'un jugement moral - brûler un chaton pour le plaisir est mal, par exemple - ne dépend en aucune manière des désirs ou des croyances de quiconque. Si, par quelque improbable concours de circonstances, tous les êtres humains en arrivaient à croire le contraire, ce jugement pourrait toujours être dit vrai; il continuerait de refléter adéquatement les faits moraux. 
Le réalisme moral a de nombreux attraits. Il permet de préserver l'objectivité de la morale, d'expliquer pourquoi les désaccords moraux prennent habituellement la forme d'une recherche de la "bonne réponse ", ou encore de rendre compte de la possibilité de progrès moral. Toutefois, les adversaires de cette position ont maints reproches à lui adresser, notamment sur les plans ontologique et épistémique. Quelle peut bien être la nature des faits moraux? Comment pourrions-nous avoir accès cognitivement à de tels faits?

Récemment, certains philosophes ont suggéré qu'il y aurait une tension entre le réalisme moral et l'hypothèse fort probable selon laquelle l'évolution aurait joué un rôle déterminant dans le façonnement de la moralité1. L'être humain, comme toutes les autres créatures, est le produit de l'évolution. Il a par conséquent été soumis à toutes sortes de pressions évolutionnistes qui ont contribué à le façonner tant physiquement (pouces opposables, station verticale, etc.) que psychologiquement (émotions, cognition, etc.). De nombreuses recherches récentes permettent de croire que certaines tendances évaluatives que nous associons à la morale, prendre soin de nos enfants ou encore rendre service en retour à celui qui nous a rendu service, pourraient avoir pour origine de telles pressions ${ }^{2}$. C'est-à-dire que ceux parmi nos ancêtres qui avaient ces tendances évaluatives auraient bénéficié d'un avantage par rapport aux autres sur le plan reproductif. Or il semble y avoir une coïncidence remarquable entre un grand nombre de jugements moraux que nous estimons être vrais et ceux qui auraient eu une grande valeur sélective. Le problème qui se pose pour le réalisme moral est de rendre compte de la relation entre les pressions évolutionnistes et les faits moraux.

Sharon Street formule ce problème sous la forme d'un dilemme pour les réalistes ${ }^{3}$. Suivant ce qu'elle nomme «dilemme darwinien », les réalistes peuvent soit nier, soit affirmer l'existence d'un lien entre les pressions évolutionnistes et les faits moraux.

S'ils optent pour la première branche du dilemme, les réalistes sont contraints d'affirmer que les pressions évolutionnistes nous ont poussés dans toutes sortes de directions, et ce sans égard à la vérité évaluative. Il semble pour le moins improbable qu'un mécanisme aussi aléatoire soit susceptible de produire des jugements moraux reflétant des vérités évaluatives indépendantes. Si l'on rejette l'idée qu'un heureux hasard nous a permis de les atteindre, il faut alors conclure que la plupart de nos jugements moraux sont probablement faux puisqu'ils sont "contaminés» par l'influence des pressions évolutionnistes. Un tel résultat est difficile à réconcilier avec le réalisme moral puisqu'il mène à la conclusion que, bien qu'il existe des faits

1. Voir notamment Ruse et Wilson, I986; Street, 2006 et Joyce, 2006.

2. Par exemple Sober et Wilson, I998; Haidt et Joseph, 2004; De Waal, 2005 et Kitcher, $20 I$ I.

3. Street, 2006. 
moraux, nous n'en avons vraisemblablement aucune connaissance. Comme le remarque Shafer-Landau, cette position sceptique, bien qu'elle soit logiquement cohérente, est fort peu attrayante pour les réalistes ${ }^{4}$.

S'ils optent plutôt pour la seconde branche du dilemme, les réalistes doivent alors expliquer la nature de la relation, c'est-à-dire expliquer comment les pressions évolutionnistes ont pu nous faire tendre de manière systématique vers la vérité évaluative. Selon Street, il n'existe aucun compte rendu adéquat, sur le plan scientifique, de cette présumée relation.

De nombreux auteurs réalistes se sont efforcés de répondre au dilemme darwinien depuis sa parution. Les stratégies argumentatives qu'ils adoptent varient considérablement. Certains choisissent de s'attaquer à la prémisse du dilemme, soit l'idée que l'évolution ait influencé de manière déterminante nos jugements moraux ${ }^{5}$. D'autres optent pour la seconde branche du dilemme, en élaborant par exemple ce que David Enoch nomme des explications par un troisième facteur ${ }^{6}$. Enfin, certains auteurs adoptent la première branche du dilemme stipulant qu'il n'existe aucune relation particulière entre les pressions évolutionnistes et les faits moraux.

C'est ce dernier type de solution qui retiendra notre attention dans ce qui suit. Je soumettrai à un examen critique une stratégie que je nommerai, suivant la terminologie introduite par Shafer-Landau, la réponse naturelle (natural reply) ${ }^{7}$. Elle consiste, pour l'essentiel, à identifier des jugements épistémiquement fiables et exempts d'influence évolutionniste à partir desquels nous puissions corriger les jugements soi-disant «contaminés» par l'évolution. Si la stratégie obtient le succès escompté, les réalistes pourront maintenir qu'il nous est possible de connaître les faits moraux, et ce même si l'évolution ne permet pas de les "pister» (track). La version la plus complète de la réponse naturelle a été élaborée par Peter Singer et Katarzyna de LazariRadek, dans un article intitule The Objectivity of Ethics and the Unity of Practical Reason ${ }^{8}$ et reprise dans leur plus récent livre, The Point of View of the Universe ${ }^{9}$. C'est cette version qui servira d'exemple afin d'illustrer les problèmes auxquels fait face cette approche.

Deux objections principales seront formulées. Une première objection est d'ordre épistémologique. L'intuitionnisme philosophique, sur lequel s'appuient les auteurs, fait face à des problèmes qui paraissent difficiles à surmonter. Notons que cette objection a une portée limitée dans la mesure où

4. Shafer-Landau, 20I2, p. I.

5. Nagel, 20 I2.

6. Parmi les auteurs ayant opté pour cette stratégie, mentionnons Copp, 2008; Wielenberg, 20I0; Skarsaune, 20II, Brosnan, 20II et Enoch, 20II.

7. Shafer-Landau, 2012.

8. De Lazari-Radek et Singer, 20 I 2.

9. De Lazari-Radek et Singer, 20I4. Notons que les auteurs n'ont pas recours au terme «réponse naturelle pour décrire leur approche. Néanmoins, elle possède toutes les caractéristiques distinctives de cette stratégie argumentative telle que la décrit Shafer-Landau. 
elle ne concerne que la proposition particulière de Singer et de Lazari-Radek. On pourrait imaginer une version de la réponse naturelle ne recourant pas à cette épistémologie douteuse. La seconde objection, plus fondamentale, est que leur solution ne permet pas de rendre compte de manière satisfaisante de la coïncidence entre de nombreux jugements moraux que nous estimons être vrais et ceux que l'adoption d'une perspective évolutionniste nous permet d'anticiper. Toutes les versions possibles de la réponse naturelle s'exposent à cette objection, raison pour laquelle elle devrait être abandonnée. C'est du moins ce que je tenterai de montrer.

\section{Une version de la réponse naturelle: Singer et de Lazari-Radek}

Il convient de présenter d'abord plus en détail en quoi consiste la solution proposée par Singer et de Lazari-Radek. Un premier élément à noter est que leur approche est en continuité avec la position qu'ils défendent sur le plan de l'éthique normative, soit une forme d'utilitarisme. Singer défend la thèse forte selon laquelle nous devrions (toujours) faire ce qui est préférable pour le bien-être de tous. Il s'est notamment fait connaître pour avoir soutenu que chacun a le devoir de donner une portion substantielle de ses revenus à des étrangers dans le besoin s'il en a les moyens ${ }^{10}$. Pour appuyer ses thèses, il a souvent eu recours à des arguments évolutionnistes. Dans «Ethics and Intuitions ", par exemple, il suggère que les intuitions morales typiquement déontologiques auraient souvent pour origine des réactions émotives façonnées par les pressions évolutionnistes, tandis que les intuitions typiquement conséquentialistes seraient davantage cognitives ou raisonnées ${ }^{11}$. Ces dernières seraient par conséquent plus fiables épistémiquement ${ }^{12}$.

Cela nous permet de constater que Singer et de Lazari-Radek souscrivent à la prémisse du dilemme darwinien selon laquelle l'évolution aurait exercé une influence majeure sur nos attitudes évaluatives ${ }^{13}$ et à la conclusion selon laquelle cela remettrait en cause le statut épistémique de nombreux jugements moraux. Or la question importante est de savoir jusqu'où les arguments darwiniens qui sont déployés autorisent le scepticisme ${ }^{14}$. C'est notamment sur la réponse qu'ils apportent à cette question que Singer et de

\section{Singer, 1972 .}

11. Singer semble douter que l'on puisse encore parler d'intuition dans ce cas. Il propose le terme « intuition rationnelle " (rational intuition) pour les distinguer des autres types d'intuitions et il les compare aux axiomes rationnels mis de l'avant par Sidgwick. Singer, 2005, 35035I. Voir aussi Greene, 2003 et Greene, 2013.

12. La conclusion selon laquelle les intuitions conséquentialistes seraient plus fiables que les intuitions déontologiques est sévèrement critiquée par Selim Berker. Plus généralement, Berker estime qu'aucune conclusion normative ne peut être dérivée des études empiriques (imagerie par résonance magnétique par exemple) visant à déterminer la manière dont nous parvenons à certains jugements. Voir Berker, 2009.

13. De Lazari-Radek et Singer, 20I4, p. I79.

14. Singer, I98 I, p.72 et Kahane, 20I I, p. I I4. 
Lazari-Radek se distinguent de Street. Pour les premiers, seule l'objectivité d'une classe relativement restreinte de jugements moraux (les jugements "déontologiques» en particulier) est menacée, tandis que pour la seconde, c'est l'objectivité de l'ensemble du domaine évaluatif qui l'est. Pour soutenir cette position, Singer et de Lazari-Radek invoquent une faculté permettant de reconnaître le statut privilégié de certains jugements moraux, soit la raison. Bien entendu, il s'avère nécessaire de fournir une explication de la manière dont cette faculté aurait pu évoluer et des raisons qui nous incitent à croire qu'elle nous permet de reconnaître des vérités morales indépendantes.

Les auteurs suggèrent que le jugement moral est un sous-produit de la faculté de raisonner. L'une des raisons qu'ils ont d'envisager les choses ainsi est qu'ils s'accordent avec Street pour affirmer qu'il n'y aurait pas le moindre avantage, en matière de succès reproductif, à percevoir des vérités morales indépendantes. Toutefois, la raison — qui suppose la capacité de déduire des conclusions à partir de prémisses, de planifier les moyens à mettre en œuvre afin d'atteindre certaines fins, etc. - offre des avantages manifestes aux créatures qui en bénéficient. Dans la mesure où elle permet aux humains de résoudre quantité de problèmes pouvant affecter leur survie, la raison a pu faire l'objet d'une sélection. Une fois qu'on est doté d'une telle faculté, il devient difficile d'accepter des jugements moraux reposant sur des bases arbitraires ou, inversement, de rejeter des jugements reposant sur des bases solides, et ce même si ceux-ci ne contribuent aucunement à la survie ou à la reproduction, voire les compromettent ${ }^{15}$. Le fait de parvenir à certains jugements moraux désavantageux est en quelque sorte le prix à payer pour pouvoir bénéficier des autres avantages conférés par la raison. L'élimination de la tendance à les formuler aurait signifié l'élimination de la raison, celle-ci étant indivisible ${ }^{16}$. Autrement dit, «la moralité est un corollaire inévitable d'une intelligence utile du point de vue de l'évolution: en devenant des animaux rationnels, les êtres humains, eo ipso, sont devenus des créatures dotés d'un sens moral ${ }^{17}$ ».

Le principe de bienveillance, qui stipule que chacun est moralement tenu d'accorder autant d'importance à son propre bien qu'à celui d'autrui ${ }^{18}$, constitue un bon exemple d'un principe fondé en raison ne présentant aucun avantage du point de vue de l'évolution. Tous les mécanismes évolutionnaires qui sont habituellement avancés pour expliquer les dispositions

15. De Lazari-Radek et Singer, 20I2, p. I6.

16. "A plausible explanation of the existence of these capacities is that the ability to reason comes as a package that could not be economically divided by evolutionary pressures ", de Lazari-Radek et Singer, 20I 2, p. I7.

17. McGinn, I979, p. 93.

18. «Each one is morally bound to regard the good of any other individual as much as his own, except in so far as he judges it to be less, when impartially viewed, or less certainly knowable or attainable by him ", Sidgwick, Methods of Ethics, cité dans de Lazari-Radek et Singer, 20I 2, p. I7. 
altruistes chez les humains ont une étendue relativement limitée. La sélection de parentèle permet d'expliquer le souci envers ceux qui nous sont apparentés génétiquement; l'altruisme réciproque, le souci envers ceux qui sont en position de nous rendre la pareille; la sélection de groupe, le souci envers le groupe d'appartenance (et la méchanceté à l'égard des autres groupes), etc. Par conséquent, il est difficile de voir quel mécanisme, outre la raison, pourrait être à l'origine de l'idée voulant que l'on doive accorder une considération égale aux intérêts de parfaits étrangers ${ }^{19}$.

Notons que le principe de bienveillance n'est pas, ni ne peut être envisagé par Singer et de Lazari-Radek comme une simple extension aux étrangers des formes d'altruisme restreintes favorisées par la sélection naturelle. En effet, ces points de départ évaluatifs ont, de l'avis des auteurs, une base arbitraire. Or il est difficile de concevoir comment une extension raisonnée d'un principe d'altruisme partiel et arbitraire pourrait nous conduire à la vérité d'un principe d'altruisme universel. Comme le remarque Guy Kahane, nous risquerions d'être comme une personne qui "rencontre un fou qui compte les brins d'herbe dans sa cour et qui lui dit que, la distinction entre sa cour et celle d'un autre étant arbitraire, il devrait plutôt compter les brins d'herbe partout dans le monde ${ }^{20}$ ». Reconnaissant la validité de cette objection, les auteurs en appellent plutôt au fait que le principe de bienveillance soit évident-en-soi. C'est-à-dire que tout être rationnel devrait en arriver à la conclusion que, du point de vue de l'univers, son bien n'importe pas davantage que celui d'un autre, sans qu'il faille pour cela s'appuyer sur quelque point de départ évaluatif dont il serait par ailleurs doté.

Le fait que: I) la réflexion rationnelle mène à la conviction que l'intuition est évidente-en-soi; 2) que plusieurs penseurs y soient parvenus de façon indépendante; et 3) qu'aucune explication évolutionniste ne puisse être fournie pour en rendre compte sont autant de considérations qui militent en faveur de l'idée selon laquelle il s'agirait d'une vérité morale indépendante ${ }^{21}$. De manière générale, toute «intuition" ou jugement qui résisterait à ce test devrait être jugée plus fiable qu'un jugement ne satisfaisant qu'un, deux, voire aucun de ces critères. C'est sur cette base, par exemple, que Singer et de Lazari-Radek concluent que le principe de bienveillance devrait avoir préséance sur le principe de l'égoïsme rationnel, «résolvant» ainsi le problème du dualisme de la raison pratique formulé

19. De Lazari-Radek et Singer, p. 2I et de Lazari-Radek et Singer, 20I4, I8 5-I 87.

20. Cité dans de Lazari-Radek et Singer, 20I4, p. I9I.

21. de Lazari-Radek et Singer, 20I2, p. 26 et de Lazari-Radek, 20I4, p. I95. ShaferLandau propose quant à lui trois critères différents permettant de reconnaître que des jugements moraux peuvent servir «d'outils non contaminés» (untainted benchmarks) à partir desquels évaluer d'autres jugements, soit: I) ils sont exempts d'influence évolutionniste; 2) nous pouvons savoir quels sont ces jugements; et 3 ) ils sont vraisemblablement hautement justifiés (warranted) (Shafer-Landau, 20I 2, p. 6) 
autrefois par Sidgwick ${ }^{22}$. En effet, l'égoïsme rationnel, qui stipule que chacun devrait tendre à maximiser son propre bien, est sujet à une explication évolutionniste et serait, par conséquent, épistémiquement moins fiable que le principe de bienveillance ${ }^{23}$.

Même s'ils se bornent à la défense du principe de bienveillance, les auteurs estiment que d'autres principes ou intuitions pourraient satisfaire ces trois critères et, par conséquent, être des candidats plausibles au statut de vérités morales ${ }^{24}$.

\section{Querelle d'interprétation}

Un dernier point doit être clarifié avant de développer nos objections. Nous avons soutenu que la réponse naturelle était une manière de répondre au dilemme darwinien en optant pour la première branche du dilemme darwinien, selon laquelle il n'existe aucun lien entre pressions évolutionnistes et faits moraux. Or Singer et de Lazari-Radek affirment à quelques reprises que leur proposition adopte plutôt la seconde branche du dilemme, selon laquelle il existerait un tel lien ${ }^{25}$. Notre interprétation nous paraît nettement préférable dans la mesure où elle respecte davantage les paramètres établis par Street dans la formulation du dilemme ${ }^{26}$.

La seconde branche du dilemme suppose d'établir une relation entre l'influence exercée par l'évolution sur nos attitudes évaluatives et la reconnaissance de certaines vérités morales indépendantes. De manière générale, une caractéristique fera l'objet d'une sélection si elle augmente la valeur sélective (fitness) d'un organisme ${ }^{27}$. Par exemple, le fait de pouvoir reconnaître les

22. Quoi que nous n'en ayons pas fait mention jusqu'ici, l'un des objectifs principaux des auteurs, outre le fait de répondre aux critiques darwiniennes contre l'objectivité de la morale, consiste à résoudre le problème du dualisme de la raison pratique en faveur de l'impartialité. Le problème en question réside dans la tension entre deux axiomes rationnels (bienveillance universelle/égoïsme rationnel) prescrivant parfois des conduites contraires. Sidgwick concluait The Methods of Ethics sur une note peu optimiste quant aux chances de le résoudre. Puisque, contrairement à l'égoïsme rationnel, le principe de la bienveillance universel n'est pas sujet à une explication évolutionniste, les auteurs estiment qu'il devrait avoir préséance.

23. de Lazari-Radek et Singer, 20I4, p. I97.

24. de Lazari-Radek et Singer, 20I4, p. I96.

25. "Either there is no relationship between the evolution of the basic capacity and the independent moral truths — in which case it is a remarkable coincidence that the basic capacity had, as a by-product, a capacity to grasp objective moral truths- or there is some relationship between the evolved basic capacity and the capacity to grasp independent moral truths. We have taken the second horn of this dilemma ", de Lazari-Radek et Singer, 20I2, p. I8. Voir aussi de Lazari-Radek et Singer, 20I4, p. I82.

26. Voir en particulier Street, 2006, p. I42-I44.

27. Ce principe général admet des exceptions. Certains comportements peuvent être sélectionnés qui requièrent de sacrifier la vie de l'individu qui agit. De tels comportements n'ont de toute évidence aucune valeur sélective pour l'individu, mais ils augmentent habituellement la valeur sélective d'autres individus qui lui sont apparentés. On parlera alors de valeur sélective inclusive. Voir W. D. Hamilton, I964; Sober et Wilson, I998. 
éléments de son environnement immédiat ou de pouvoir dénombrer des objets peut favoriser le succès reproductif des individus. Si l'on envisage les vérités physiques ou mathématiques abstraites auxquelles la raison nous donne accès comme une extension de ces capacités de base, nous pouvons à bon droit affirmer qu'il existe un lien entre les pressions évolutionnistes et les vérités mathématiques ou physiques indépendantes ${ }^{28}$. Or, la proposition des auteurs, en ce qui concerne la morale, n'a pas du tout cette forme. L'explication qu'ils proposent de l'émergence de la raison ne dépend en aucune manière du pistage des vérités morales. Au contraire, l'objectif de leur hypothèse est précisément de rendre compte de l'apparition d'une faculté permettant de les reconnaître, en dépit du fait que la perception de ces vérités ne présente aucun avantage en matière de succès reproductif. Le fait que la raison puisse par la suite les percevoir n'est pas suffisant pour que l'on puisse affirmer l'existence d'un lien entre les pressions évolutionnistes et les vérités morales indépendantes. Il semble plus juste, par conséquent, d'affirmer qu'elle adopte la première branche du dilemme.

Cette précision est importante dans la mesure où la suggestion selon laquelle ils auraient adopté la seconde branche du dilemme laisse croire, à tort, que la coïncidence remarquable évoquée ci-dessus - selon laquelle les vérités morales indépendantes correspondent largement aux jugements qu'une perspective évolutionniste nous permet d'anticiper - a reçu une explication satisfaisante. Puisque le recours au hasard constitue le principal élément nous incitant à douter de la plausibilité de leur solution, nous insistons pour que ce fait ne soit pas perdu de vue, quelle que soit la manière dont on décide par ailleurs de résoudre cette querelle d'interprétation ${ }^{29}$.

\section{Deux objections}

Les principales raisons que nous avons de douter de la plausibilité de la solution proposée par Singer et de Lazari-Radek sont: I) qu'elle repose sur une épistémologie douteuse, et 2) qu'elle ne permet pas d'expliquer autrement que par un heureux hasard le fait que les jugements moraux que nous estimons être vrais soient précisément ceux vers lesquels l'évolution nous aurait fait tendre. Le premier problème pourrait en principe être évité par les réalistes puisqu'il est sans doute possible de développer une version de la

28. La possibilité de développer une telle généalogie pour ces croyances peut même être envisagée comme une condition essentielle du succès du dilemme darwinien et, plus généralement, de toute critique évolutionniste du réalisme moral. Sans le "pistage » de ces vérités, les arguments, scientifiques ou autres, avancés dans le dilemme auraient eux-mêmes une origine douteuse. Selon Railton, l'argument darwinien risquerait alors de s' "enfoncer dans le même sol où il avait au préalable enfoncé la moralité » (Railton, 2000, p. 57). C'est sans doute la raison pour laquelle tous les critiques darwiniens insistent sur l'asymétrie entre le cas de la science et celui de la morale. Par exemple Street, 2006; Sinclair, 2006; et Joyce, 2006, p. I83.

29. Afin de simplifier la présentation du texte, nous présenterons nos arguments en sousentendant toujours que nous nous situons sur la première branche du dilemme. 
réponse naturelle ne recourant pas à l'intuitionnisme philosophique ou à toute autre forme d'épistémologie fondationaliste. Cependant, le second problème se pose nécessairement lorsque l'on adopte la première branche du dilemme darwinien (niant l'existence d'un lien entre les pressions évolutionnistes et les vérités évaluatives), comme le fait la réponse naturelle. Ce problème constitue par conséquent une raison majeure de douter des chances de succès de cette stratégie argumentative, et ce, quelle que soit la manière dont elle est déployée.

\section{Une épistémologie douteuse}

Commençons par l'objection d'ordre épistémologique. Le recours à des principes évidents-en-soi, bien qu'il s'inscrive dans une vénérable tradition philosophique, est problématique à plusieurs égards. Essentiellement, Singer et de Lazari-Radek adoptent l'intuitionnisme philosophique tel qu'il fut autrefois défendu par Sidgwick. Selon cette position, les principes moraux ultimes, et seulement ces principes, seraient évidents-en-soi et ils seraient justifiés de manière non inférentielle.

L'un des principaux attraits de la théorie est qu'elle permet en principe d'éviter une régression à l'infini dans la justification des jugements moraux. L'argument "classique» soutient que, pour toute croyance (morale ou autre), il est possible d'exiger une justification. Quelles que soient les raisons qui seront invoquées pour l'appuyer, on pourra demander ce qui justifie ces raisons, et ainsi de suite ad infinitum ${ }^{30}$. Puisque l'on ne parvient jamais à arrêter la régression, nos croyances sont toujours en mal de justification ${ }^{31}$. L'intérêt d'un principe moral évident-en-soi est qu'il permet d'interrompre la chaîne justificative puisqu'il n'a pas lui-même à être justifié. Selon Sidgwick, quatre conditions doivent être remplies pour que des principes puissent avoir ce statut: I) ils doivent être clairs et précis, 2) être évidents après réflexion, 3) être mutuellement compatibles et, enfin, 4) être généralement acceptés ${ }^{32}$. Singer et de Lazari-Radek proposent, comme nous l'avons $\mathrm{vu}$, des critères légèrement différents permettant de vérifier la fiabilité d'un principe. L'ajout le plus notable est sans doute le critère selon lequel ce principe ne devrait pas se prêter à une explication évolutionniste ou à toute autre explication qui en ferait le résultat d'un processus ne permettant pas de pister la vérité.

Le fondationalisme, dont l'intuitionnisme philosophique est l'une des déclinaisons possibles, a fait l'objet de maintes critiques, et un examen

30. Pour une présentation formelle de «l'argument classique» en faveur de l'intuitionnisme, voir Pekka Väyrynen, 2008, p. 49I. Pour une défense de l'idée selon laquelle l'argument de la régression à l'infini est inadéquat, voir entre autres Heath, 20II, p. I37-I52; SayreMcCord, 20I3, p. II4-I I 8.

31. Brink, I988; Sinnott-Armstrong, 2006; Väyrynen, 2008.

32. Brink, I988, p. 294. 
détaillé de chacune d'entre elles dépasse largement les ambitions du présent commentaire. Nous nous contenterons d'un survol de quelques arguments qui nous semblent décisifs, ce qui devrait suffire à se faire une idée des obstacles que doivent surmonter les adeptes de cette théorie. Considérant que même ses défenseurs doutent que l'on puisse prouver l'existence de propositions évidentes-en-soi ${ }^{33}$, il semble légitime que ses adversaires ne soient pas davantage obligés d'en réfuter l'existence hors de tout doute.

Comme nous l'avons spécifié, les réalistes moraux ne sont pas tenus d'adopter une épistémologie intuitionniste. Les arguments qui seront présentés ne peuvent par conséquent suffire à discréditer toutes les versions possibles de la réponse naturelle. Néanmoins, le "mariage» entre le réalisme moral non naturaliste - la principale cible du dilemme darwinien - et une épistémologie intuitionniste est courant dans la littérature, et il est, selon certains, particulièrement bien justifié. Cette combinaison de thèse a été défendue entre autres par Russ Shafer-Landau, Michael Huemer et Robert Audi $^{34}$. Étant donné sa relative "popularité ", il s'avère pertinent d'explorer les difficultés, majeures à notre avis, que présente cette position.

\section{La justification non inférentielle}

La principale raison qui motive notre scepticisme envers l'intuitionnisme concerne la nature de la justification. Affirmer qu'une croyance est évidenteen-soi, c'est affirmer qu'elle s'auto-justifie. Le seul fait de considérer attentivement et de comprendre une telle proposition serait suffisant pour qu'il soit justifié d'y croire ${ }^{35}$. Cela suppose notamment que l'on puisse être entièrement justifié d'adhérer à une croyance, même en l'absence de toute capacité à l'inférer à partir d'autres croyances ${ }^{36}$. Plusieurs doutent qu'une croyance, qu'elle soit morale ou non, puisse avoir cette propriété ${ }^{37}$.

Comme le souligne Brink, "aucune croyance à propos du monde ne peut être aussi la raison pour laquelle on estime que cette croyance est vraie». L'auto-justification peut être envisagée comme étant "l'instance extrême (limiting case) du raisonnement circulaire, c'est-à-dire que l'autojustification est le plus petit cercle justificatif imaginable ${ }^{38} »$. Pour justifier une croyance $p$, on invoque généralement des croyances de second ordre au sujet de $p$, à propos, par exemple, du type de croyance qu'est $p$ et de ce qui fait qu'une telle croyance a des chances d'être vraie ${ }^{39}$. C'est à un tel exercice

33. «I don't believe that it is possible to prove or demonstrate the existence of such propositions. The best that can be done is to offer candidates that are appealing (if any are), and to reply to criticisms of the idea " Shafer-Landau, 2003, p. 247.

34. Bedke, 2009, p. I88-г89.

35. Audi, I999, p. 206; Shafer-Landau, 2003, p. 247.

36. Sinnott-Armstrong, 2006, p. 342.

37. BonJour, I985; Brink, I989; Sinnott-Armstong, 2006.

38. Brink, I989, p.I 6 .

39. Ibid., p. I I6-I I7. 
de justification que Singer et de Lazari-Radek se livrent lorsqu'ils énumèrent certaines propriétés du principe de bienveillance, notamment le fait qu'il n'ait pas une origine évolutionniste, que nous y parvenions par le raisonnement, etc. Par conséquent, on ne saurait affirmer que ce principe s'autojustifie.

Bien entendu, il est possible de rejeter cette conclusion en soutenant que la proposition peut être connue de manière inférentielle, d'où cet exercice de justification, et de manière non inférentielle. C'est-à-dire que les raisons qui sont avancées ne font qu'ajouter à la justification que possède déjà la proposition en raison de son évidence; il s'agit d'une proposition épistémiquement surdéterminée (epistemically overdetermined $)^{40}$.

Walter Sinnott-Armstrong rejette cette possibilité. S'appuyant sur des travaux récents en psychologie et en neurosciences, il suggère que nos croyances morales requièrent toujours une justification inférentielle. Il identifie cinq principes, courants dans nos pratiques épistémiques, à partir desquels nous pouvons déterminer qu'une croyance nécessite une confirmation indépendante, soit: I) lorsque l'individu qui possède la croyance (believer) est partial; 2) lorsqu'il existe un désaccord et qu'aucune raison indépendante ne permet de préférer une croyance plutôt qu'une autre; 3) lorsque des émotions sont susceptibles d'influencer le jugement; 4) lorsque les circonstances peuvent conduire à des illusions; et 5) lorsqu'une croyance a une origine dont la fiabilité est douteuse.

Or les croyances morales sont particulièrement susceptibles d'être influencées de l'une ou l'autre de ces manières, et ce même si cette influence n'est pas toujours aisément perçue par l'agent qui les possède. Par exemple, les travaux de Haidt et de Greene ont permis d'amasser quantité de preuves de l'influence (pernicieuse) que peuvent avoir les émotions sur le jugement moral (principe 3$)^{41}$; des études ont montré que l'ordre de présentation des options, et d'autres facteurs n'ayant vraisemblablement aucune pertinence morale, peuvent affecter le jugement ${ }^{42}$ (principe 4); des facteurs culturels ou évolutionnistes peuvent expliquer que nous parvenions à un jugement donné sans qu'il soit nécessaire d'invoquer la vérité de ce jugement ${ }^{43}$ (principe 5), etc.

Considérant cela, il s'avère indispensable, pour qu'un jugement moral soit justifié, de montrer qu'il n'est pas influencé de cette manière ou, qu'en dépit de cette influence, des raisons indépendantes permettent de le justifier. Une telle démonstration requiert inévitablement l'inférence de croyances à partir d'autres croyances. Par conséquent, toute justification qu'un principe

40. «Self-evident beliefs might thus be epistemically overdetermined-inferentially and non-inferentially justifiable ", Shafer-Landau, 2003, p. 248. Voir aussi Audi, I999, p. 216-2 I 7.

41. Greene et Haidt, 2002; Moll et al., 200I; Wheatley et Haidt, 2005.

42. Unger, I996; Kahneman et Tversky, I979.

43. Street, 2006. 
moral pourrait avoir en vertu de sa seule «évidence » est extrêmement faible, voire inexistante ${ }^{44}$.

\section{Comment reconnaître l'évidence-en-soi ?}

Les défenseurs de l'intuitionnisme soutiendront probablement que ces considérations sont insuffisantes, qu'elles ne permettent pas de démontrer qu'il n'existe aucune proposition évidente-en-soi. On peut se demander quelles conditions permettraient de le faire. Le flou considérable entourant la procédure à suivre afin de déterminer si une croyance est bel et bien évidenteen-soi ${ }^{45}$ constitue un autre problème important qui afflige l'intuitionnisme. La théorie menace de devenir infalsifiable.

Comme le souligne Bedke, les défenseurs de l'intuitionnisme ont investi beaucoup d'efforts afin de déterminer ce que l'évidence-en-soi n'est pas, négligeant parfois la tâche positive de définir ce qu'elle est. Ainsi, il ne serait pas nécessaire qu'une proposition évidente-en-soi ne puisse être défaite par d'autres considérations; des modes de justification non intuitifs pourraient augmenter ou diminuer le support envers une proposition évidenteen-soi; il ne serait pas non plus nécessaire que l'on puisse voir l'évidence d'une telle proposition; il serait possible de la comprendre adéquatement sans toutefois y souscrire, etc ${ }^{46}$. Il est difficile de voir dans ces circonstances quelles sont les conditions permettant de réfuter une allégation d'évidenceen-soi. Le fait qu'elles soient difficiles à réfuter ne constitue pas, à proprement parler, un argument contre l'intuitionnisme ${ }^{47}$. Néanmoins, on peut constater le caractère ad hoc de toutes ces stipulations. Elles ont toutes les apparences de conditions ajoutées au fil du temps en réponse aux attaques des adversaires de la théorie.

\section{Une solution de rechange: le cohérentisme}

Le recours à des principes évidents-en-soi permettrait aux réalistes d'établir la base la plus solide qui soit afin de contrer les distorsions introduites par les pressions évolutionnistes, soit des jugements moraux fondationels ${ }^{48}$. Cependant, nous avons suggéré que l'appel à l'évidence est problématique et qu'il devrait être abandonné. Pour s'acquitter de la tâche, peut-être les réalistes peuvent-ils se contenter de croyances qui, sans être fondationelles, sont tout de même «considérées " (considered moral beliefs).

44. Sinnott-Armstrong, 2006.

45. «I have also yet to see a reliable test for determining whether a proposition is selfevident ", Väyrynen, 2008, p. 506.

46. Bedke, 2009, p. I93-I94.

47. Väyrynen, 2008 , p. 506.

48. Encore faudrait-il que tous les jugements moraux vrais puissent être dérivés à partir de ces quelques jugements fondationels, ce qui constitue un autre problème "classique " que doivent résoudre les intuitionnistes (Shafer-Landau, 20I 2, p. 6). 
Certains réalistes rejettent le fondationalisme au profit d'une épistémologie cohérentiste ${ }^{49}$. Selon cette position, "une croyance $p$ est justifiée si elle fait partie d'un système de croyances cohérent et que la cohérence de $p$ explique au moins en partie pourquoi l'on croit $p^{50}$ ». Les défenseurs du cohérentisme ${ }^{51}$ insistent habituellement sur le rôle important que jouent les jugements moraux considérés ${ }^{52}$ dans le processus de justification. Ces jugements, bien que faillibles et révisables, jouiraient d'une crédibilité initiale suffisante pour leur permettre de servir de point de départ à la réflexion rationnelle . $^{53}$.

Il existe un certain nombre d'objections dirigées contre la combinaison entre une épistémologie cohérentiste et le réalisme moral. Par exemple, plusieurs doutent que la cohérence interne d'un système de croyances offre une raison de croire que ces croyances reflètent des vérités indépendantes. Si l'on suppose que des systèmes de croyances différents peuvent être (maximalement) cohérents, on doit admettre que tous ne peuvent être vrais simultanément. C'estl'objection des systèmes alternatifs cohérents ${ }^{54}$. Si le fondationalisme est inadéquat et que le cohérentisme est incompatible avec le réalisme moral ${ }^{55}$, nous disposons d'un argument de taille contre le réalisme moral, et ce indépendamment du succès du dilemme darwinien. Défendre une telle hypothèse exigerait cependant d'examiner quantité d'autres arguments et cela nous éloignerait des considérations pertinentes du point de vue du dilemme darwinien. Voyons quelles sont ces considérations.

Ce qui importe pour le défenseur de la réponse naturelle, c'est de pouvoir identifier des jugements exempts d'influence évolutionniste et jouissant d'une grande crédibilité initiale. Shafer-Landau ${ }^{56}$ propose quelques candidats,

49. Brink, I989.

50. Ibid., p. I06.

51. Rawls, I97I; Brink, I989, p. I36; Sayre-McCord, 20I3.

52. Sayre-McCord parle plutôt de croyance permissivement justifiée (permissively justified belief).

53. " $[\mathrm{R}]$ eliance on considered moral beliefs is an important part of moral justification. Moral theories are tested by their coherence with, among other things, considered moral beliefs [...]. The reliability of these considered moral beliefs is explained by psychological theories about cognitive reliability and generally plausible moral theories whose credibility is more or less independent of the particular moral theories being tested. Considered moral beliefs, though revisable, have initial credibility", Brink, I989, p. I36.

54. Timmons, I990, p. IOI.

55. L'idée que le réalisme moral et le fondationalisme soient incompatibles a aussi été défendue par certains auteurs (voir Bedke, 2009).

56. Shafer-Landau défend une épistémologie fondationaliste et non cohérentiste. Cependant, son article examine les stratégies argumentatives que peuvent adopter les réalistes, quelles que soient leurs allégeances épistémologiques. Les jugements invoqués ici ne sont sans doute pas envisagés par Shafer-Landau comme étant des exemples de propositions évidentes-en-soi, mais ils correspondent assez bien à l'idée que l'on peut se faire de "jugements considérés". Quoi qu'il en soit, rien de ce qui suivra ne dépend du choix de ces jugements particuliers plutôt que d'autres. 
dont la considération pour les générations futures, le souci envers les petits animaux ou encore la compassion envers les étrangers. Selon lui, il serait nécessaire pour celui qui avance une critique darwinienne de pouvoir montrer que toutes ces croyances sont le produit d'influences évolutionnistes ${ }^{57}$.

Il n'est pas impossible qu'un certain nombre des jugements invoqués puissent se prêter, directement ou indirectement, à une explication évolutionniste. Le souci envers les petits animaux offre un exemple éclairant. On peut d'abord douter que la "petitesse» soit un facteur déterminant: les serpents, les cafards, les araignées, et quantité d'autres petits animaux souvent jugés effrayants ou nuisibles ne suscitent sans doute pas le même "souci » que les chatons, les chiots ou les blanchons. La peur, voire la phobie, d'animaux tels les serpents et les araignées ${ }^{58}$, tout comme l'attachement aux petits animaux de compagnie, que manifestent presque tous les enfants ${ }^{59}$, ont reçu des explications évolutionnistes convaincantes. La valeur sélective de la «joliesse»(cuteness) a aussi reçu ce genre d'explication: le fait que les humains estiment que leurs enfants sont mignons constitue un bon incitatif à leur prodiguer tendresse et soins. Cette attitude serait susceptible d'être étendue au-delà des bornes de l'espèce, en particulier envers les bébés animaux $^{60}$. S'ajoutent à cela certains facteurs contingents. Le fait d'avoir ou non possédé un animal de compagnie, par exemple, peut influencer l'attitude que l'on développe envers les animaux ${ }^{61}$. Par conséquent, on peut douter que l'adhésion à un tel jugement soit une indication qu'il existe des vérités morales indépendantes ou qu'il puisse servir à corriger d'autres jugements «contaminés».

Peut-être pourrions-nous produire une explication semblable pour les autres jugements avancés par Shafer-Landau. Ces explications risqueraient toutefois de n'être que des histoires ad hoc. Plutôt que de tenter de le faire, nous suggérerons que l'idée même selon laquelle une telle démonstration serait requise est erronée. En effet, elle repose sur une compréhension inadéquate du dilemme darwinien. En aucun temps Street ne suggère que tous nos jugements moraux sont le produit des pressions évolutionnistes. Ils peuvent aussi résulter d'une influence culturelle, de la réflexion rationnelle ou d'une combinaison de ces facteurs ${ }^{62}$. Par voie de conséquence, on voit mal ce qui justifierait que le défenseur d'une critique darwinienne soit tenu de prouver que toutes les croyances apparemment désavantageuses soient en réalité

57. «[I]n order to entirely fend off the Natural Reply, debunkers must provide an indirect explanation of the origins of every moral belief that is both highly presumptively warranted and apparently maladaptive. Debunkers are very far from having done this ", Shafer-Landau, 20I 2, p. 7-8.

58. Öhman, 2009.

59. Prokop et al., 20 Iо.

60. Morreall, I99I.

61. Prokop et al., 20 Iо.

62. ., Street, 2006, p. I I3-II 4 . 
issues de pressions évolutionnistes. Ce qu'il importe de constater, c'est que, même si l'on concède que ce n'est pas le cas, les réalistes n'ont toujours pas montré que les jugements qu'ils invoquent sont bel et bien justifiés, ni qu'ils correspondent en quelque manière à des vérités morales indépendantes. À une époque pas si lointaine, les jugements selon lesquels "les sorcières doivent être brûlées» ou «les esclaves désobéissants doivent être battus» apparaissaient probablement à ceux qui les défendaient comme jouissant d'une grande crédibilité prima facie. On peut effectivement douter que de tels jugements aient pour origine des pressions évolutionnistes. Cela offret-il le moindre support à la thèse des réalistes ?

Sans doute ceux-ci pourront-ils répondre que ces jugements particuliers n'étaient pas justifiés et qu'ils ne reflétaient que les croyances erronées qui avaient cours à cette époque. Nous avons considérablement progressé depuis ce temps, et les jugements que nous affirmons sont plus sûrs. Encore faut-il en faire la démonstration. Le critère proposé par Shafer-Landau a pour effet de transférer le fardeau de la preuve sur les épaules des critiques darwiniens sans raison valable. Pourquoi ne reviendrait-il pas aux réalistes de montrer: I) que tous les jugements "non contaminés » qu'ils invoquent ne sont pas le produit d'influences évolutionnistes ou de tout autre processus (la culture notamment) ne permettant pas de pister la vérité; et 2) que la réflexion rationnelle à partir de ces jugements suffit à corriger les distorsions introduites par l'évolution? Pour reprendre la formule de Shafer-Landau, les réalistes sont encore «très loin d'avoir fait cela ${ }^{63}$ ».

\section{Une seconde objection: le problème de la coïncidence}

Nous avons jusqu'ici considéré des arguments ciblant uniquement certaines versions possibles de la réponse naturelle. Afin de montrer que toutes les versions possibles de cette stratégie échouent, un argument de portée plus générale s'avère nécessaire. Nous appellerons cet argument le problème de la coïncidence. Il stipule qu'au moins deux coïncidences majeures sont nécessaires pour que les faits se soient déroulés comme le proposent les défenseurs de la réponse naturelle. La première coïncidence notable est qu'une capacité aussi sophistiquée que celle qui permet d'identifier des vérités morales indépendantes soit le résultat d'un processus n'entretenant pas le moindre rapport avec ces vérités ${ }^{64}$. La seconde coïncidence, peut-être plus étonnante encore, est que les jugements moraux que plusieurs endossent après mûre réflexion - ceux qui semblent être des candidats au statut de vérités morales indépendantes - reflètent pour une large part des attitudes évaluatives ayant une grande valeur sélective.

Ce problème est, pour l'essentiel, déjà identifié par Street lorsqu'elle expose les résultats qui attendent ceux qui choisiraient d'opter pour la

63. Shafer-Landau, 2012 , p. 8.

64. Street, 2006, p. I42-I 44 . 
première branche du dilemme ${ }^{65}$. Notre intention dans ce qui suit est de montrer que la réponse naturelle ne parvient pas à l'éviter. L'argument n'a pas la prétention de réfuter hors de tout doute cette stratégie, mais plutôt de montrer qu'une fois ce problème pris en considération, cette stratégie apparaît comme la moins attrayante des options disponibles.

La plupart des réalistes concéderaient probablement que plusieurs jugements moraux qu'ils estiment être vrais correspondent à ceux qu'une perspective évolutionniste permet d'anticiper. Par exemple, il est plausible que le fait de venir en aide à un proche, de préférence à un étranger, ou de favoriser son groupe d'appartenance soient des attitudes évaluatives ayant fait l'objet d'une sélection. Nombreux sont les réalistes moraux qui s'efforcent d'intégrer de telles "intuitions» à leurs théories normatives. Dans "The Fragmentation of Value», Thomas Nagel reconnaît les obligations spéciales que nous avons envers nos proches, notre communauté ou notre pays comme étant des considérations morales importantes ${ }^{66}$. Brink avance la même idée lorsqu'il suggère que l'utilitarisme objectif qu'il défend "accorde une grande valeur au développement de relations personnelles impliquant souci réciproque et engagement. Un souci approprié [...] pour le bien-être de ses proches, par conséquent, limitera la quantité de bienveillance que [l'utilitarisme objectif] requiert de la part des agents ${ }^{67}{ }^{\prime}$. On pourrait aisément multiplier les exemples.

Si l'on s'en tient à la première branche du dilemme, comme le font les tenants de la réponse naturelle, il faut admettre que les pressions évolutionnistes ne tendent pas de manière systématique vers les vérités évaluatives indépendantes. Ne reste que le hasard pour expliquer un tel alignement entre les vérités évaluatives et les tendances évaluatives ayant une grande valeur sélective. Cela ne constitue pas en soi un motif suffisant pour rejeter la proposition. D'heureux hasards se produisent parfois et, en l'absence d'une meilleure explication, peut-être qu'un appel à la coïncidence serait satisfaisant. David Enoch formule ce constat de la façon suivante:

Des corrélations brutes, inexpliquées et même inexplicables ne sont pas, après tout, impossibles et donc, même si le réaliste n'a aucune explication à offrir pour expliquer la corrélation entre les vérités normatives et nos croyances normatives, [...] le réalisme n'a pas encore été réfuté. Seulement, nous devrions opter pour la meilleure théorie métaéthique, celle qui [...] explique le mieux ce qui doit être expliqué. Donc, si le réaliste ne peut expliquer une corrélation frappante comme celle sur laquelle insiste le défi épistémologique, le réalisme perd des points de plausibilité, peut-être au point d'être inacceptable ${ }^{68}$.

65. Ibid., p. I 2 I-I 25.

66. «There are five fundamental types of value [...]. First, there are specific obligations to other people or institutions: obligations to patients, to one's family, to the hospital or university at which one works, to one's community or one's country ", Nagel, I979, p. I29.

67. Brink, I989, p. 267.

68. Enoch, 20II, p. I65. 
Pour éviter de s'en remettre entièrement au hasard, il est possible pour les réalistes de soutenir que la correspondance entre les vérités morales indépendantes et les attitudes évaluatives ayant une grande valeur sélective est moins grande qu'il y paraît. Plusieurs philosophes, après tout, rejettent les intuitions décrites précédemment au profit d'une conception plus impartiale de ce que requiert la morale. C'est notamment le cas de Singer et de LazariRadek lorsqu'ils défendent le principe de la bienveillance universelle. Les influences évolutionnistes qui donnent naissance à des formes d'altruisme plus limitées sont considérées sans équivoque par ces auteurs comme autant de «sources de contamination» du jugement moral. Dans « Ethics and Intuitions", Singer affirme d'ailleurs qu'une théorie normative ne devrait pas chercher à accommoder nos intuitions, à plus forte raison si leur origine, évolutionniste ou autre, les rend suspectes ${ }^{69}$.

Ce genre d'argument introduit toutefois de nouvelles complications. En dissociant complètement l'évolution de tendances altruistes, chez les humains, de la vérité morale indépendante que serait le principe de bienveillance, Singer et de Lazari-Radek ne font que "déplacer» la coïncidence à un autre niveau. C'est-à-dire que même en supprimant la correspondance entre les pressions évolutionnistes et les vérités morales, il demeure une correspondance non moins frappante entre les pressions évolutionnistes et les concepts moraux. Les auteurs supposent que les tendances altruistes dont l'évolution nous aurait dotés au moyen de divers mécanismes (sélection de parentèle, altruisme réciproque, etc.) ne contribuent en aucune manière à expliquer pourquoi l'altruisme universel est bien. Eussions-nous évolué de telle manière que nous n'ayons aucune de ces tendances, l'évolution de la raison et de son corollaire, le raisonnement moral, nous aurait tout de même rendus capables de reconnaître un principe comme celui de l'altruisme universel. Nous sommes donc devant le constat suivant: d'une part, des pressions évolutionnistes qui n'entretiennent pas le moindre rapport avec la vérité du principe d'altruisme universel font évoluer une faculté, la raison, nous permettant de découvrir cette vérité. D'autre part, des dispositions altruistes qui semblent étroitement liées avec l'idée que nous nous faisons de la moralité évoluent par toutes sortes de mécanismes, mais cela ne contribue en rien à expliquer pourquoi l'altruisme universel est bien. Au contraire, cela contamine notre jugement et tend à nous éloigner de la vérité morale. Comment expliquer cela?

On pourrait reprendre le même raisonnement pour les idées de justice, de réciprocité, de blâme ou encore de récompense. On peut présumer que toute théorie morale souhaitera se prononcer sur ces sujets. Si l'on envisage les vérités indépendantes sur ces questions comme ne devant absolument rien aux pressions évolutionnistes, comme étant un pur produit de l'activité rationnelle, on risque de reproduire le même résultat. Singer reconnaît qu'un 
système de récompense et de punition peut être envisagé comme une conséquence (lointaine) de l'altruisme réciproque, ou encore que le fait de distinguer ceux qui font preuve de réciprocité de ceux qui n'en font pas preuve puisse mener à la formation d'une proto-notion (crude notion) de ce que sont l'équité (fairness) et la tricherie ${ }^{70}$. À moins que le théoricien ne soit prêt à redéfinir les contours de la moralité de manière à exclure complètement de telles notions, il semble que nous trouverons toujours un lien étroit entre les tendances évaluatives façonnées par l'évolution et les principaux concepts auxquels nous reconnaissons la moralité. Est-il plausible que l'évolution ait conduit les humains à développer des attitudes évaluatives pouvant être associées à toutes les classes importantes de jugements moraux (sexualité, hiérarchie, équité, altruisme, etc.), mais que ces attitudes ne doivent (presque) jamais être prises en considération dans la détermination de ce qui est bien ou mal?

Il semble que d'autres explications possibles soient moins mystérieuses. Les antiréalistes, dans une certaine mesure, n'ont pas à résoudre le problème de la cö̈ncidence, puisqu'il ne se pose pas à partir de leur perspective. Puisqu'ils rejettent l'existence de vérités morales indépendantes, l'idée que les attitudes évaluatives façonnées par l'évolution correspondent ou non à de telles vérités leur est étrangère. Quant à la coïncidence entre ces attitudes et les concepts moraux, elle s'explique tout simplement par le fait que les jugements moraux que nous endossons après réflexion sont une fonction des attitudes évaluatives à partir desquelles s'est amorcée la réflexion. À notre avis, une théorie de ce type devrait être préférée à une théorie réaliste. Cependant, même en demeurant dans un cadre réaliste, de meilleures explications semblent disponibles. En adoptant plutôt la seconde branche du dilemme darwinien, la correspondance entre attitudes évaluatives et vérités morales pourra n'avoir que l'apparence d'une coïncidence. C'est ce que cherchent à accomplir les explications par un troisième facteur, une stratégie argumentative déployée par de nombreux auteurs réalistes, notamment David Copp, Knut Olav Skarsaune, Eric Wielenberg et David Enoch ${ }^{71}$. Comme l'indique Shafer-Landau, si l'on ignore tout du magnétisme, le fait que deux aimants s'attirent ou se repoussent de façon systématique peut s'apparenter à une incroyable coïncidence. Cette impression disparaîtra une fois que nous connaîtrons les mécanismes sous-jacents. Le même raisonnement s'applique en ce qui concerne la moralité ${ }^{72}$.

Un trait essentiel de ce type de réponse est qu'elle requiert l'existence d'un lien entre les pressions évolutionnistes et les vérités morales indépendantes. La correspondance n'a toutefois pas à être parfaite. Le réaliste pourra toujours, s'il le souhaite, maintenir que le principe de bienveillance

70. Singer, 2005 .

71. Pour une critique des explications par un troisième facteur, voir Street, 2008.

72. Shafer-Landau, 20I 2, p.IO. 
est une vérité morale indépendante. Il suffira de montrer que l'évolution nous a permis de développer des tendances altruistes qui sont suffisamment près de la vérité évaluative pour que la raison nous permette par la suite de dériver ce principe. Si l'on souhaite aller plus loin et couper entièrement les ponts entre pressions évolutionnistes et moralité, comme semblent vouloir le faire Singer et de Lazari-Radek, il faudra accepter que de nombreuses coïncidences demeurent inexpliquées et, probablement, inexplicables.

\section{Conclusion}

En définitive, que l'on suppose l'alignement ou le non-alignement entre les attitudes évaluatives façonnées pas l'évolution et les vérités morales indépendantes, la réponse naturelle doit adopter l'un ou l'autre des scénarios où figurent les curieux hasards décrits précédemment. Cela fait perdre, selon l'expression d'Enoch, des "points de plausibilité ${ }^{73}$ " à toute réponse de ce type. Nous laisserons ouverte la question de savoir si cela suffit à la rendre inacceptable. Notons cependant que d'autres hypothèses sont disponibles qui permettent, du moins à première vue, une explication plus satisfaisante du phénomène. L'hypothèse de Singer et de Lazari-Radek, et possiblement toute autre version de la réponse naturelle qui ne serait pas supplémentée par un quelconque mécanisme de "pistage " des vérités morales, peinent à soutenir la comparaison. C'est-à-dire que, même en adoptant l'hypothèse charitable selon laquelle les réalistes auraient en main des «jugements considérés » permettant de corriger les distorsions introduites par l'évolution, la proposition échouerait tout de même en raison des coïncidences inexpliquées qui subsisteraient. La possibilité pour les réalistes de répondre adéquatement au dilemme darwinien semble donc dépendre de l'adoption de la seconde branche du dilemme.

\section{Bibliographie}

Audi, R. "Self-Evidence », Nô̂s, 33(I3), I999, 205-228.

Bedke, M. S. "Intuitive Non-Naturalism Meets Cosmic Coincidence », Pacific Philosophical Quarterly, 90(2), 2009, I 88-209.

Berker, S. "The Normative Insignificance of Neuroscience », Philosophy \& Public Affairs, 37(4), 2009, 293-329.

BonJour, L. The Structure of Empirical Knowledge, New York, Cambridge University Press, I985.

Brink, D. O. "Sidgwick's Dualism of Practical Reason ", Australasian Journal of Philosophy 66(3), I988, 29I-307.

- Moral Realism and the Foundations of Ethics, New York, Cambridge University Press, I989.

Brosnan, K. «Do the Evolutionary Origins of our Moral Beliefs Undermine Moral Knowledge? ", Biology \& Philosophy, 26(I), 20I I, 5 I-64. 
Copp, D. «Darwinian Skepticism about Moral Realism», Philosophical Issues, I 8(I), 2008, I 86-206.

de Lazari-Radek, K. et P. Singer. "The Objectivity of Ethics and the Unity of Practical Reason ", Ethics, I 23(I), 2012, 9-3I.

. The Point of View of the Universe, Oxford, Oxford University Press, 2014 .

De Waal, F. Our Inner Ape, New York, The Berkley Publishing Group, 2005.

Enoch, D. Taking Morality Seriously: a Defense of Robust Realism, New York, Oxford University Press, $20 \mathrm{II}$.

Greene, J. "From Neural 'Is' to Moral 'Ought': What Are the Moral Implications of Neuroscientific Moral Psychology?", Nature Reviews Neuroscience 4(10), 2003, 846-850.

- Moral Tribes: Emotion, Reason, and the Gap between Us and Them, New York, Penguin, 2013.

Greene, J. et J. Haidt. «How (and Where) Does Moral Judgment Work? », Trends in Cognitive Sciences, 6(1 2), 2002, 517-523.

Haidt, J. et C. Joseph. «Intuitive Ethics: How Innately Prepared Intuitions Generate Culturally Variable Virtues ", Daedalus, I33(4), 2004, 55-66.

Hamilton, W. D. "The Genetical Evolution of Social Behaviour, II ", Journal of Theoretical Biology, 7(I), I964, I7-52.

Heath, J. Following the Rules: Practical Reasoning and Deontic Constraint, New York, Oxford University Press, 20I I.

Joyce, R. The Evolution of Morality, Cambridge, The MIT Press, 2006.

Kahane, G. «Evolutionary Debunking Arguments ", Noûs, 45(I), 20I I, IO3-I 25.

Kahneman, D. et A. Tversky. "Prospect Theory: An Analysis of Decision under Risk ", Econometrica: Journal of the Econometric Society, I979, 263-29I.

Kitcher, P. The Ethical Project, Cambridge, Harvard University Press, $201 \mathrm{I}$.

McGinn, C. "Evolution, Animals, and the Basis of Morality ", Inquiry, 22(I-4), I979, 8I-99.

Moll, J., P. J. Eslinger et R. d. Oliveira-Souza. «Frontopolar and Anterior Temporal Cortex Activation in a Moral Judgment Task: Preliminary Functional MRI Results in Normal Subjects ", Arquivos de Neuro-Psiquiatria, 59(3B), 200I, 657-664.

Morreall, J. "Cuteness ", The British Journal of Asthetics, 3 I(I), I99I, 39-47.

Nagel, T. I979. Mortal Questions: Canto, New York, Cambridge University Press, I99I.

. Mind and Cosmos: Why the Materialist Neo-Darwinian Conception of Nature is almost Certainly False, New York, Oxford University Press, 2012.

Öhman, A. "Of Snakes and Faces: An Evolutionary Perspective on the Psychology of Fear ", Scandinavian Journal of Psychology, 50(6), 2009, 543-552.

Prokop, P. et S. D. Tunnicliffe. «Effects of Having Pets at Home on Children's Attitudes toward Popular and Unpopular Animals ", Anthrozoos: A Multidisciplinary Journal of The Interactions of People \& Animals, 23(I), 2010, 2I-35.

Railton, P. «Darwinian Building Blocks », Journal of Consciousness Studies 7( I-2), 2000, I-2.

Rawls, J. I971. A Theory of Justice, Cambridge, Belknap Press, I999.

Ruse, M. et E. O. Wilson. "Moral Philosophy as Applied Science», Philosophy, 6I(236), I986, I73-I92. 
Sayre McCord, G. "Coherentism and the Justification of Moral Beliefs ", Ethical Theory: an Anthology, R. Shafer-Landau, Wiley. com, I3, 2012.

Shafer-Landau, R. Moral Realism: a Defence, New York, Oxford University Press, 2003.

. "Evolutionary Debunking, Moral Realism and Moral Knowledge », Journal of Ethics \& Social Philosophy 7, I-I, $20 \mathrm{I} 2$.

Sinclair, N. "Two Kinds of Naturalism in Ethics ", Ethical Theory and Moral Practice, 9(4), 2006, 4I7-439.

Singer, P. «Famine, Affluence, and Morality», Philosophy \& Public Affairs, I(3), I972, 229-243.

- I98 I. The Expanding Circle. Princeton, Princeton University Press, 20 I I.

—. "Ethics and Intuitions ", The Journal of Ethics, 9(3-4), 2005, 33 I-352.

Sinnott-Armstrong, W. "Moral Intuitionism Meets Empirical Psychology», Metcethics After Moore, T. Horgan et M. Timmons, Oxford University Press, 2006.

Skarsaune, K. O. "Darwin and Moral Realism: Survival of the Iffiest ", Philosophical Studies, I $52(2), 201$ I, 229-243.

Sober, E. et D. S. Wilson. Unto Others: The Evolution and Psychology of Unselfish Behavior, Cambridge, Harvard University Press, 1998.

Street, S. "A Darwinian Dilemma for Realist Theories of Value ", Philosophical Studies I 27(I), 2006, I09-I66.

- «Reply to Copp: Naturalism, Normativity, and the Varieties of Realism Worth Worrying About ", Philosophical Issues, I 8(I), 2008, 207-228.

Timmons, M. «On the Epistemic Status of Considered Moral Judgments ", The Southern Journal of Philosophy, 29(SI), I990, 97-I 29.

Unger, P. K. Living High and Letting Die: Our Illusion of Innocence, New York, Cambridge University Press, I996.

Väyrynen, P. «Some Good and Bad News for Ethical Intuitionism », The Philosophical Quarterly, 58(232), 2008, 489-5I I.

Wheatley, T. et J. Haidt. "Hypnotic Disgust Makes Moral Judgments more Severe ", Psychological Science, I6(10), 2005, 780-784.

Wielenberg, E. J. "On the Evolutionary Debunking of Morality ", Ethics, I2O(3), 20I0, 44I-464. 